Artigo

\title{
Propuesta de Método de Cálculo de Presión para un Modelo de Intensidad de Potencial
}

\author{
José Carlos Fernández Alvarez ${ }^{1}$ (D), Oscar Díaz Rodríguez ${ }^{2}$ (D), Albenis Pérez Alarcón ${ }^{1}$ \\ ${ }^{1}$ Instituto Superior de Tecnologías y Ciencias Aplicadas, Departamento de Meteorología, \\ Universidad de La Habana, La Habana, Cuba. \\ ${ }^{2}$ Instituto de Meteorología, La Habana, Cuba.
}

Recibido em: 15 de Enero de 2018 - Aceptado em: 22 de Mayo de 2018

\begin{abstract}
Resumen
Se propone un método para el cálculo de la presión mínima central en un modelo de intensidad potencial de ciclones tropicales determinado a partir del perfil radial de viento de Willoughby, lo que garantiza una relación físicamente directa entre la presión mínima central y la velocidad máxima del viento en superficie. Se observó que el modelo para los huracanes más intensos de la cuenca atlántica mantuvo una incertidumbre de un valor máximo de $6 \mathrm{hPa}$, pudiéndose apreciar valores de presión muy cercanos a la realidad. Se comprobó para los huracanes menos intensos la capacidad de simular la presión mínima central y la velocidad máxima del viento en superficie dentro de un rango muy cercano a los valores reales.
\end{abstract}

Palabras-clave: ciclones tropicales, perfil de viento, presión mínima central, intensidad potencial de huracanes.

\section{Proposal of Pressure Calculation Method for a Model of Potential Intensity}

\begin{abstract}
A method for calculating the minimum central pressure in a model of potential intensity of tropical cyclones determined from the Willoughby wind profile, which provides a physically direct relationship between the central minimum pressure and the maximum wind speed in the surface is proposed. It was observed that the model for the most intense hurricanes of the Atlantic basin maintained an uncertainty of a maximum value of $6 \mathrm{hPa}$. Values of pressure very close to the reality were noticed. It was checked the ability to simulate the minimum central pressure and the maximum surface wind speed for less intense hurricanes within very close range to the actual values.
\end{abstract}

Keywords: tropical cyclones, wind profile, central minimum pressure, potential hurricane intensity.

\section{Introducción}

Los ciclones tropicales son considerados uno de los fenómenos naturales más mortales y destructores que existen. Pueden variar significativamente de un día al siguiente, en intensidad, en estructura del ojo, en estructura en la capa fronteriza, entre otros elementos. Numerosas han sido las investigaciones para tratar de encontrar una respuesta a los factores que determinan estas diferencias y gobiernan el desarrollo futuro del sistema. Los mayores avances han ocurrido en los estudios realizados en la capa fronteriza, en la dinámica y en el papel que juega la banda espiral, así como en la interacción entre el fenómeno y el medio ambiente que lo rodea (Montgomery y Smith, 2014).

La predicción numérica de la trayectoria de ciclones tropicales ha aumentado enormemente desde las pasadas décadas, pero aún tiene poca fiabilidad el pronóstico del cambio de intensidad de la tormenta Elsberry et al. (1992). Parte del problema del pronóstico de la intensidad se debe a las desventajas de los modelos actuales para representar correctamente la dinámica que explica los procesos de intensificación en el núcleo de la tormenta.

Existen numerosas teorías para establecer una cuota máxima en la intensidad que pueden alcanzar los ciclones

Send correspondence to José Carlos Fernández Alvarez, jcfernandez@instec.cu. 
tropicales (Wang y Wu, 2004), las cuales no están exentas de limitantes de aplicación e implementación. En este aspecto, una de las teorías más aceptadas para la predicción de la intensidad de los huracanes es la Teoría de Emanuel (Emanuel, 1986), (Camp y Montgomery, 2001), en la cual se describen los principales procesos dinámicos $\mathrm{y}$ termoenergéticos considerando el ciclo energético de los mismos como un motor de Carnot, absorbiendo calor del océano y cediéndolo en la tropopausa. Sin embargo, esta teoría ha recibido numerosas críticas relacionadas con la irreversibilidad de todo proceso atmosférico. No obstante, la principal limitación es considerar el balance de viento gradiente en la capa fronteriza. De ahí que los resultados obtenidos de intensidad potencial en ocasiones sean inferiores a la intensidad real de los huracanes Smith et al. (2008).

Una forma de evadir la limitante del balance de viento gradiente en la capa fronteriza es asumir perfiles radiales de viento para los ciclones tropicales como los de: Holland (1997), Frisius-Scgönemann (2013) y Willoughby (2016).

El modelo de Emanuel (1986) para determinar la velocidad máxima realiza un cálculo basado en consideraciones termodinámicas, así el cálculo de la presión central del sistema se efectúa de forma independiente; por lo que se propone un método para el cálculo de la presión mínima central en un modelo de intensidad potencial de ciclones tropicales determinado a partir del perfil de viento de Willoughby (2006), que garantiza una relación directa con la determinación de la velocidad máxima del viento.

\subsection{Teoría de Emanuel}

Emanuel (1986) desarrolló una teoría de la intensidad potencial de los ciclones tropicales, considerando al ciclo termoenergético del huracán como el de un motor de Carnot, que extrae energía de la superficie marina y la cede en la tropopausa. Así el aire que es forzado a converger en la pared del ojo del ciclón se carga de humedad en su trayectoria hacia el centro, aumentando su energía estática húmeda, pues el transporte de calor no es causado solamente por la diferencia de temperaturas entre el aire en la capa fronteriza y la superficie del mar, la que en los trópicos suele ser menor de un grado centígrado, sino en mayor medida por la baja humedad del aire de la capa fronteriza respecto a la superficie del océano, este desequilibrio marca la tasa y la cota superior de aumento de la energía del aire. Así, el aire más húmedo lleva intrínseco una energía mayor ya que el calor específico del agua es notablemente mayor que el del aire seco. Entonces como fuente de energía potencial queda establecida la superficie marina, esta energía potencial es convertida por el ciclón en la energía cinética necesaria para balancear la disipación por fricción en el estado maduro del huracán.

\subsection{Modelo de la capa fronteriza atmosférica}

La capa fronteriza atmosférica juega un papel extremadamente importante en la dinámica del huracán. Una característica fundamental de esta, es que en ella no se cumple el balance de viento gradiente, pues la fuerza de fricción disminuye la velocidad tangencial en superficie lo que tiene como consecuencia que las fuerzas de Coriolis y centrífuga disminuyan también, provocando que la fuerza neta quede orientada hacia el centro del ciclón, lo que causa un flujo radial de aire en esta zona. Este movimiento del aire provoca que en la periferia del ciclón se produzca una subsidencia forzada para sustituir el aire que se desplaza hacia el centro. Se demostrado que la altura de la capa se reduce con la disminución del radio en la tormenta, por lo que existe una relación entre dos magnitudes (Smith y Montgomery, 2008). En esta investigación se utilizará un valor aproximado de unos 500 metros de espesor sugerido por Smith (2004). La convergencia de masa en la pared del ojo provoca una convección forzada en esta zona, por lo que la capa fronteriza no solo contribuye al transporte de masa en los bajos niveles sino también de los movimientos verticales del aire dentro del ciclón tropical (Smith y Montgomery, 2008).

\subsection{Perfil de viento en el tope de la capa fronteriza}

Bryan y Rotunno (2009) demostraron que la ausencia de balance de viento gradiente puede explicar el aumento de la intensidad de algunos organismos tropicales. Por tanto, la ausencia de balance de viento gradiente explica una gran parte de la superintensidad de estos organismos, definida como el exceso de la velocidad del viento por encima de la intensidad potencial calculada por la Teoría de Intensidad Potencial Clásica desarrollada por Emanuel (1986).

\subsubsection{Perfil radial de viento de Willoughby}

El perfil de viento de Willoughby (2006) se define por una función continua a trozos, donde la velocidad del viento aumenta como una potencia del radio desde el centro del sistema hasta una zona cercana al radio de vientos máximos (perfil interior). Desde el anillo de vientos máximos hacia la periferia de la tormenta la fuerza del viento disminuye exponencialmente (perfil exterior). La zona de transición determinada por el perfil interior y exterior se representa por una función polinomial:

$$
V=\left\{\begin{array}{l}
V_{\max }\left(\frac{r}{r_{m}}\right)^{n} \quad r \leq R_{1} \\
V_{i}\left(1-w_{1}\right)+V_{0} w_{1} \quad R_{1} \leq r \leq R_{2} \\
V_{\max }\left[(1-A) e^{\frac{-\left(r-r_{m}\right)}{x_{1}}}+A e^{\frac{-\left(r-r_{m}\right)}{x_{2}}}\right] r>R_{2}
\end{array}\right.
$$

donde $V_{\max }$ e el viento máximo en el radio de vientos máximos, $n, A, X_{1}$ y $X_{2}$ son parámetros que están asociados a 
la intensidad del huracán. Además $V_{i}$ y $V_{0}$ son las velocidades tangenciales en el radio $R_{1}$ y $R_{2}$ y el parámetro $w_{1}$ es la función de peso (Willoughby, 2006).

\section{Materiales y Métodos}

La propuesta de método de cálculo de presión mínima central para un modelo de intensidad máxima potencial de los ciclones tropicales consta de los siguientes elementos: las ecuaciones del modelo de Emanuel (1986) incorporando el modelo de la capa fronteriza, la utilización del perfil de viento de Willoughby (2006) en el tope de la capa fronteriza y el cálculo de la presión mínima central a partir de la velocidad máxima del viento en superficie.

\subsection{Zona de estudio}

El dominio utilizado se extiende desde los $55^{\circ}$ hasta $100^{\circ}$ de longitud oeste (W), y desde los $5^{\circ}$ hasta $35^{\circ}$ de latitud norte $(\mathrm{N})$, como puede ser visto en la Fig. 1. El criterio de selección de este dominio se basa en que posee un área que abarca el paso de los sistemas ciclónicos en su etapa de máximo desarrollo, condición idónea para el estudio de la intensidad potencial de los sistemas que afectan la zona del Caribe, especialmente aquellos que alcanzan su máxima intensidad en los mares adyacentes a Cuba (Alarcón, 2015).

\subsection{Casos de estudio}

Los casos de estudio seleccionados para la aplicación del modelo desarrollado fueron: los huracanes Gilbert (septiembre 1988), Mitch (octubre 1998) y Wilma (octubre 2005). El criterio de selección fue la intensidad alcanzada por estos organismos y el poco periodo de tiempo en el que ocurrieron las variaciones de intensidad. Además se tuvo en cuenta que algunos de los casos seleccionados de la cuenca del Atlántico se encuentran entre los huracanes más intensos de la historia en esta área geográfica. Siendo la máxima intensidad registrada comparable con la calculada por el modelo desarrollado. Los datos de temperatura

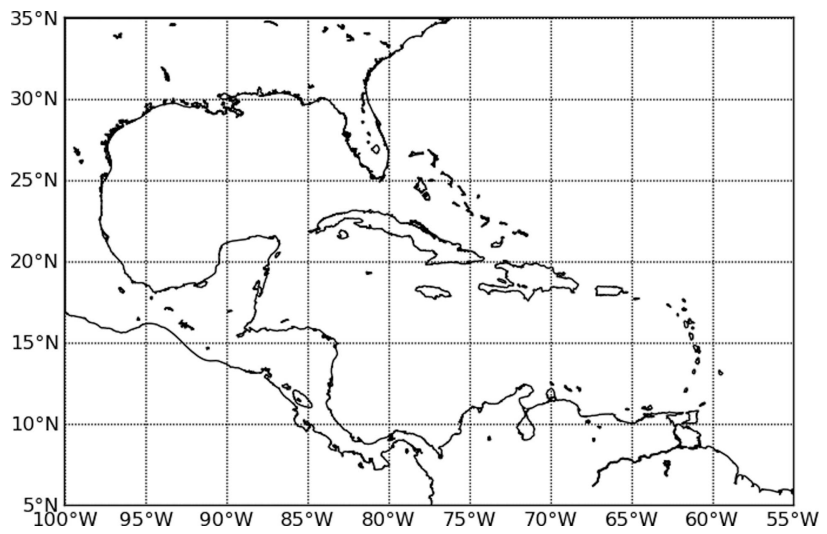

Figure 1: - Área de estudio. superficial del mar se obtuvieron del sitio web de la Administración Nacional del Océano y de la Atmósfera de los Estados Unidos (NOAA, por sus siglas en inglés) con una resolución de $0,25^{\circ}$. Los valores de presión mínima central y velocidad máxima del viento de la trayectoria del sistema se obtuvieron del sitio web del HURDAT.

\subsection{Método para el cálculo de la presión mínima central}

Teniendo en cuenta el perfil de viento de Willoughby (2006) y la ecuación para el viento gradiente:

$$
V_{g r}=\frac{-f r}{2}+\sqrt{\frac{1}{4}\left(r^{2} f^{2}\right)+\frac{r}{\rho} \frac{\partial p}{\partial r}}
$$

Se parte de considerar que el viento para cada intervalo del perfil es igual al viento gradiente. Para el primer tramo queda de la siguiente forma:

$$
V_{\max }\left(\frac{r}{r_{m}}\right)^{n}=\frac{-f r}{2}+\sqrt{\frac{1}{4}\left(r^{2} f^{2}\right)+\frac{r}{\rho} \frac{\partial p}{\partial r}}
$$

Posteriormente se despeja la derivada parcial de la presión con respecto al radio obteniéndose:

$$
\frac{\partial p}{\partial r}=\frac{\rho}{r}\left[V_{\max }\left(\frac{r}{r_{m}}\right)^{n}+\frac{f r}{2}\right]^{2}-\rho \frac{1}{4}\left(r f^{2}\right)
$$

Luego se desarrolla esta expresión y se obtiene para el intervalo $r \leq R_{1}$ la siguiente ecuación:

$$
\frac{\partial p}{\partial r}=\frac{\rho}{r} V_{\max }^{2}\left(\frac{r}{r_{m}}\right)^{2 n}+f \rho V_{\max }\left(\frac{r}{r_{m}}\right)^{n}
$$

Para el segundo intervalo $R_{1} \leq \mathrm{r} \leq R_{2}$ donde se realiza un procedimiento similar al anterior y se obtiene:

$$
\frac{\partial p}{\partial r}=\frac{\rho}{r}\left[V_{i}\left(1-w_{1}\right)+V_{0} w_{1}+\frac{f r}{2}\right]^{2}+\rho \frac{1}{4}\left(r f^{2}\right)
$$

Finalmente la ecuación para el tercer intervalo donde $\mathrm{r}>$ $R_{2}$ es:

$$
\begin{array}{r}
\frac{\partial p}{\partial r}=\frac{\rho}{r}\left[V_{\max }\left[(1-A) e^{\frac{-\left(r-r_{m}\right)}{x_{1}}}+A e^{\frac{-\left(r-r_{m}\right)}{x_{2}}}\right]+\frac{f r}{2}\right]^{2} \\
+\rho \frac{1}{4}\left(r f^{2}\right)
\end{array}
$$

donde $r$ es el radio, $r_{m}$ el radio de vientos máximos, $\rho$ la densidad de aire, $\frac{\partial p}{\partial r}$ el cambio de presión, $V_{\max }$ la velocidad máxima del viento, $f$ el parámetro de Coriolis, $R_{1}, R_{2}$, 
$A$ y $n$ constantes, $w_{1}$ el parámetro de peso y por último $x_{1}$ y $x_{2}$ son parámetros de escala (Willoughby, 2006).

\subsection{Estructura del modelo de intensidad potencial}

El modelo utiliza los ficheros NetCDF de temperatura superficial del mar (TSM) a los que se le aplica el modelo de intensidad potencial, que devuelve la velocidad máxima en superficie y la presión mínima central para luego realizar los mapas como puede ser visto en la Fig. 2.

La verificación de este se realizó a partir de aplicarle el modelo a los valores de temperaturas interpolados a los puntos de latitud y longitud de la trayectoria de los ciclones, que no se encuentran sobre tierra; para evitar los cambios bruscos de intensidad, y luego comparando los valores de presión mínima central y velocidad máxima central con los datos reales correspondientes a cada sistema como puede ser visto en la Fig. 3.

En la realización del modelo se tuvo en cuenta un procedimiento similar a Emanuel (1998) de reducir el 20\% a la velocidad máxima del viento en el tope de la capa fronteriza, y esta sería la velocidad en superficie. La relación porcentual entre ambas velocidades fue obtenida por Powell (2008) a partir de la estimación del viento máximo en superficie por mediciones en vuelos de reconocimiento en huracanes.

\subsection{Paralelización del modelo}

Se logró la paralelización computacional del modelo en el lenguaje de programación Python, lo cual permite ganar en capacidad de cómputo y disminuir el tiempo de corrida, lo que hace de esta; una herramienta eficiente y capaz de ser utilizada para investigaciones científicas

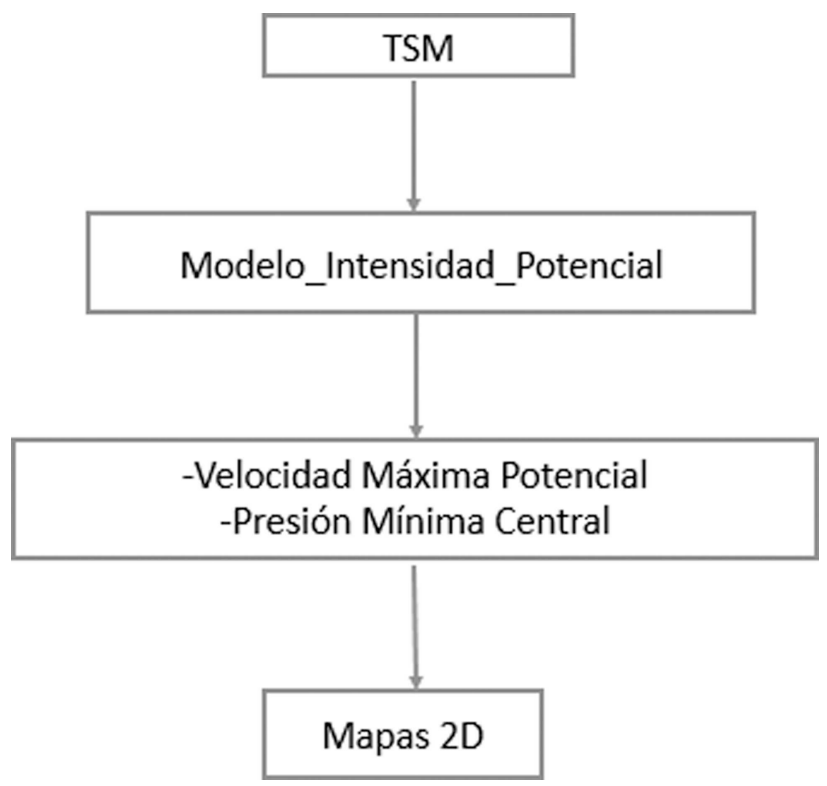

Figure 2: - Modelo de intensidad potencial.

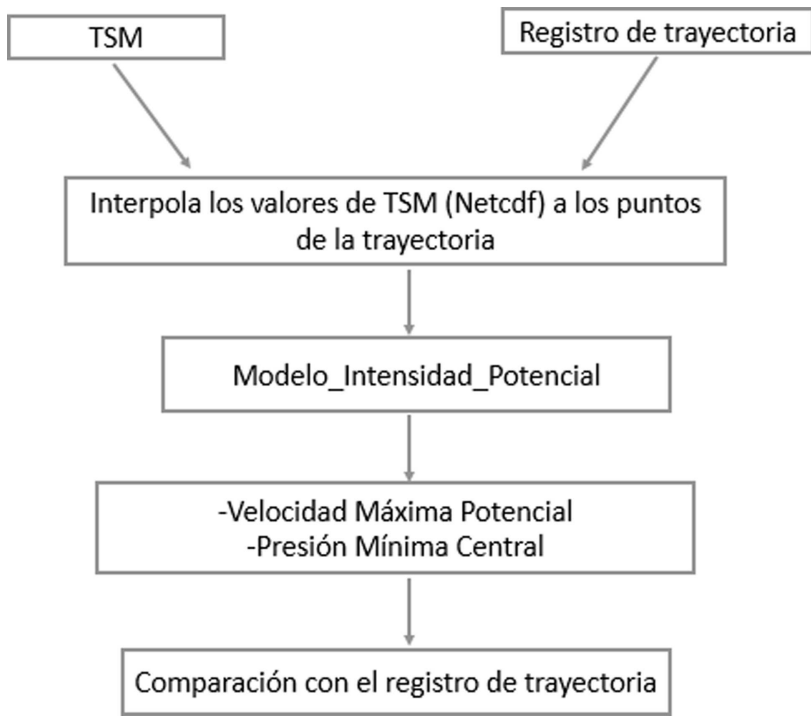

Figure 3: - Verificación del modelo de intensidad potencial.

futuras en el cálculo de la intensidad potencial de huracanes.

\section{Resultados y Discusión}

\subsection{Análisis de perfil radial de presión}

La Fig. 4 muestra los perfiles de presión de Holland (1980), Vogl (2009), el obtenido a partir del perfil de viento de Willoughby (2006) y el radio de vientos máximos para Wilma el 23 de octubre de 2005 a las 18:00 UTC. Los tres perfiles siguen un comportamiento similar hasta una distancia de $200 \mathrm{~km}$ y luego decaen rápidamente para obtener valores en el centro de sistema aproximadamente de $963 \mathrm{hPa}$, que fue la presión mínima central alcanzada para este caso de estudio. A diferencia de los perfiles de Vogl (2009) y Holland (1980) que dependen de

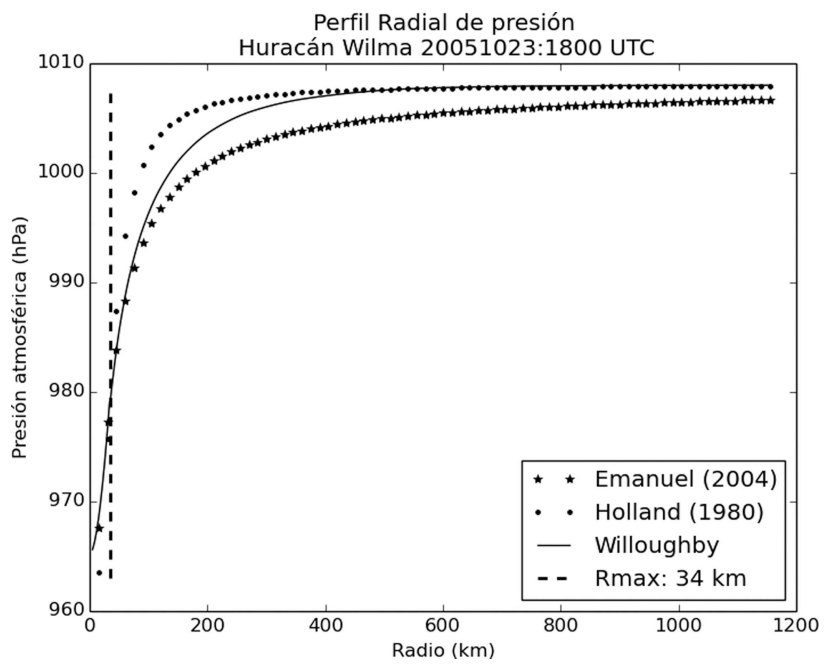

Figure 4: - Comparación de perfiles de presión para un ciclón tropical. 
la presión en el centro y en la periferia del sistema, el tercer perfil mencionado depende de la velocidad máxima del viento; por lo que permite estimar el valor de la presión mínima central. Para el caso de estudio (Wilma 20051023 18:00 UTC) el valor estimado fue de $965 \mathrm{hPa}$ con una diferencia de $2 \mathrm{hPa}$ respecto a la presión mínima real.

\subsection{Análisis de las simulaciones del modelo de intensidad potencial}

Las simulaciones realizadas en la investigación se comparan con las obtenidas para los modelo de intensidad potencial de Emanuel (1986) y de Alarcón (2015) mediante la realización de gráficos de presión mínima central y velocidad máxima del viento para la trayectoria de cada caso de estudio. Además se obtuvieron mapas para el cambio de intensidad según la evolución de cada huracán. Es necesario aclarar que los resultados alcanzados por el nuevo método de cálculo de la presión mínima central son superiores a los anteriores. Para algunos casos de estudio no se alcanzaron los resultados esperados, debido a que ciertos huracanes pueden alcanzar una intensidad; la cual no es capaz de simular el modelo. Partiendo de lo mencionado previamente se realizó una separación en huracanes más intensos, para los cuales el modelo no puede simular la presión mínima central y en menos intensos, para los que tiene la capacidad de simular esta variable.

\subsubsection{Huracanes más intensos}

3.2.1.1. Huracán Wilma. El huracán Wilma se gestó en un área de bajas presiones en la tarde del 15 de octubre a unos 120 kilómetros al sursuroeste de Montego Bay, Jamaica. La tormenta tuvo una rápida intensificación, pasando de huracán categoría 1 en la escala Saffir-Simpson a huracán categoría 5 en un plazo de 18 horas, alcanzando una presión mínima central de $882 \mathrm{hPa}$ y una velocidad máxima del viento de $296 \mathrm{~km} / \mathrm{h}$.

La corrida realizada con el modelo para este huracán permitió obtener una presión mínima central de $888 \mathrm{hPa}$ (Fig. 5) y una velocidad máxima del viento de $356 \mathrm{~km} / \mathrm{h}$ (Fig. 6), quedando $6 \mathrm{hPa}$ por encima de la presión mínima central real, lo cual puede estar relacionado con los cambios bruscos que se producen en el interior del sistema en un intervalo de tiempo de 18 horas. Posteriormente de alcanzar la intensificación máxima se observa la convergencia de las curvas como lo demuestran las Figs. 7 y 8 , esto implica que los resultados se acercan a los valores reales dentro de un rango de incertidumbre determinado.

3.2.1.2. Huracán Gilbert. El huracán Gilbert se formó a partir de una onda tropical proveniente del África, en sus inicios no tenía tanta intensidad y al moverse por el sur de Cuba, con un rumbo próximo al oeste-noroeste, sobre las aguas cálidas del Mar Caribe experimentó un proceso de rápida intensificación disminuyendo su presión central de $960 \mathrm{hPa}$ hasta $888 \mathrm{hPa}$ en 24 horas

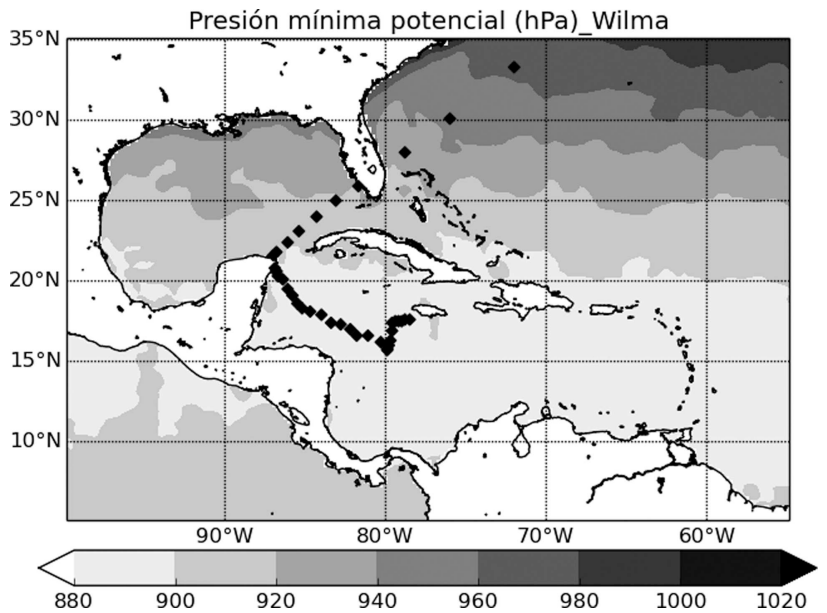

Figure 5: - Modelación de presión mínima central (Wilma).

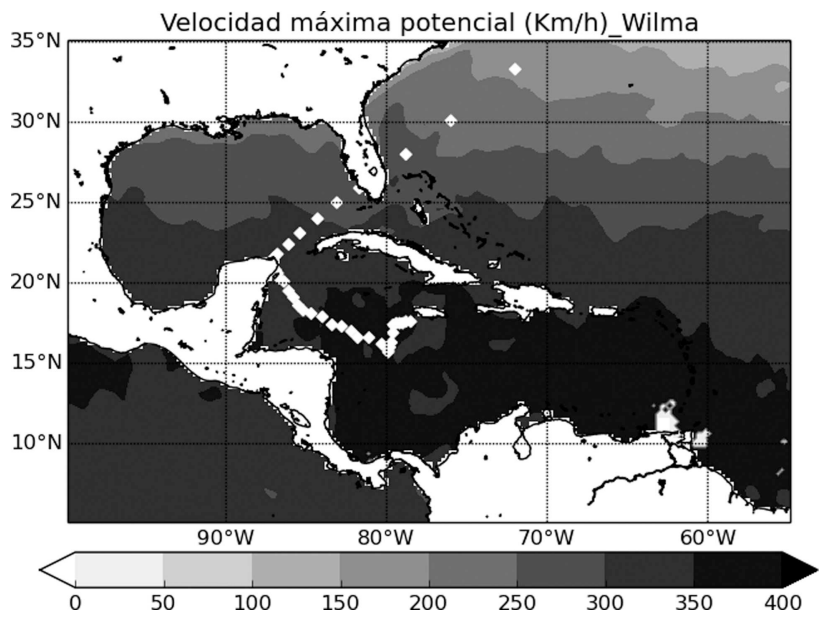

Figure 6: - Modelación de la velocidad máxima del viento (Wilma).

representando $72 \mathrm{hPa}$ en ese intervalo de tiempo y aumentando la velocidad máxima de los vientos hasta $296 \mathrm{~km} / \mathrm{h}$.

La simulación realizada con el modelo para este sistema, simuló dentro de un rango de incertidumbre cercano a la realidad, valores de presiones centrales menores (Fig. 9) y velocidades del viento mayores en superficie que las del propio huracán (Fig. 10), exceptuando los puntos de latitud y longitud donde alcanzó la máxima intensificación producto al cambio brusco de presión en un intervalo de 24 horas, el cual no puede representar el modelo por las propias limitaciones de la teoría de Emanuel (1986).

El valor de la presión central correspondiente a la simulación realizada fue de $894 \mathrm{hPa}$ (Fig. 10) siendo mayor en valor a la desarrollada por el sistema en $6 \mathrm{hPa}$ y el de la velocidad máxima del viento fue de $346 \mathrm{~km} / \mathrm{h}$ (Fig. 9). En las Figs. 11 y 12 se puede apreciar cómo a medida que la tormenta se intensificó se fue aproximando a su intensidad máxima potencial y luego de alcanzar la madurez el sistema, se aprecia la convergencia de las curvas. 


\subsubsection{Huracanes menos intensos}

3.2.2.1. Huracán Mitch. El huracán Mitch se formó a partir de una onda tropical proveniente del África a unos $95 \mathrm{~km}$ al noroeste de Cartagena, Colombia y a $670 \mathrm{~km}$ al sur de Kingston, Jamaica. Se mantuvo con un lento movimiento de traslación hacia el oeste antes de alcanzar su máxima intensidad. En este período el ojo del huracán se movió paralelo a la costa de Nicaragua y Honduras, esto condicionó que el sistema no alcanzara la máxima intensidad potencial pues su lento movimiento ocasionó que la temperatura superficial del mar disminuyera por las intensas precipitaciones. Además, dada la cercanía del centro de la tormenta a las costas de Honduras y Nicaragua, este sufrió un debilitamiento debido al efecto de la fricción con

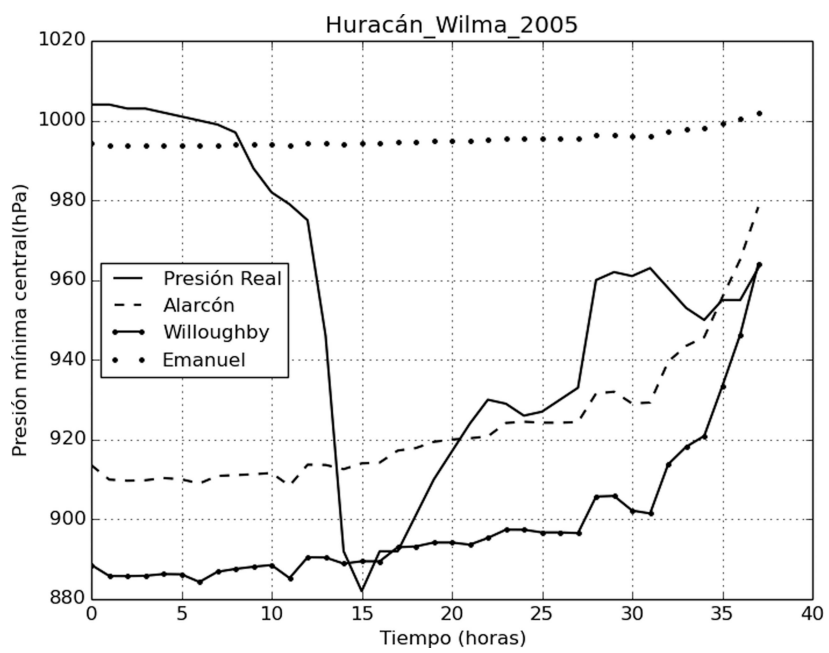

Figure 7: - Comparación entre la presión mínima real y la simulada (Wilma).

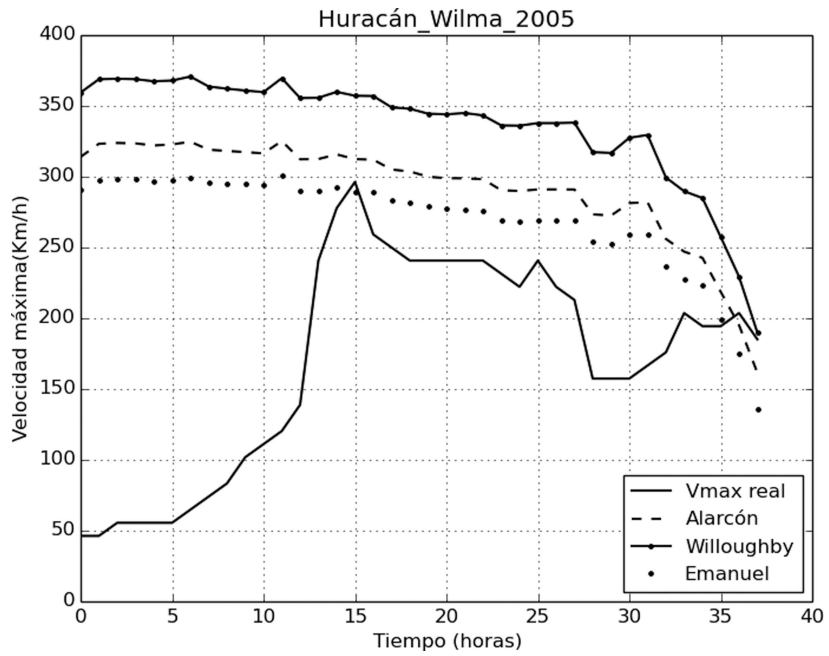

Figure 8: - Comparación entre la velocidad máxima del viento real y la simulada (Wilma).

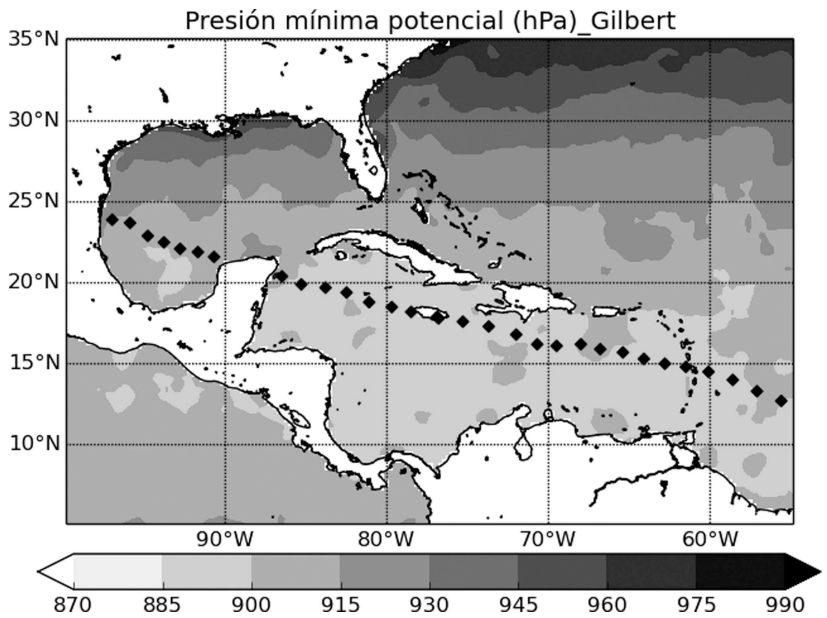

Figure 9: - Modelación de presión mínima central (Gilbert).

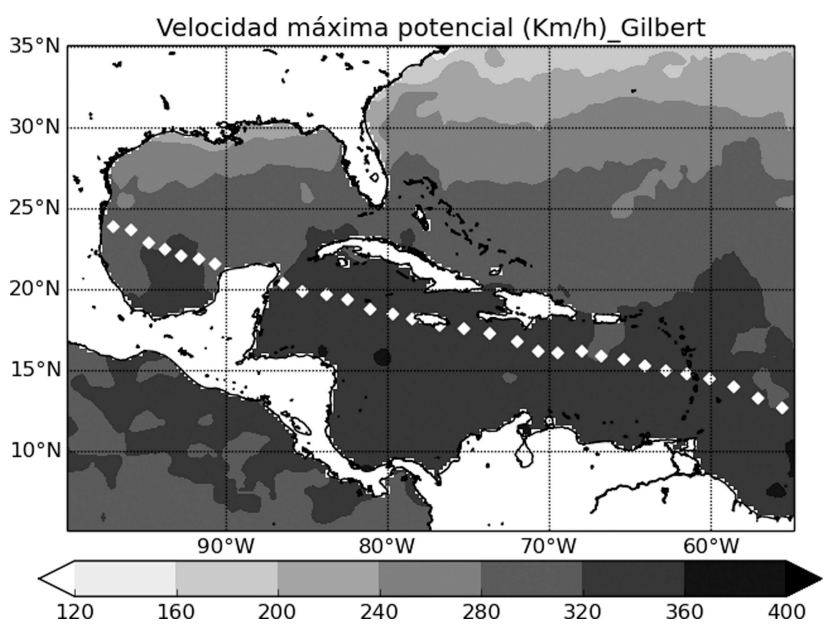

Figure 10: - Modelación de la velocidad máxima del viento (Gilbert).

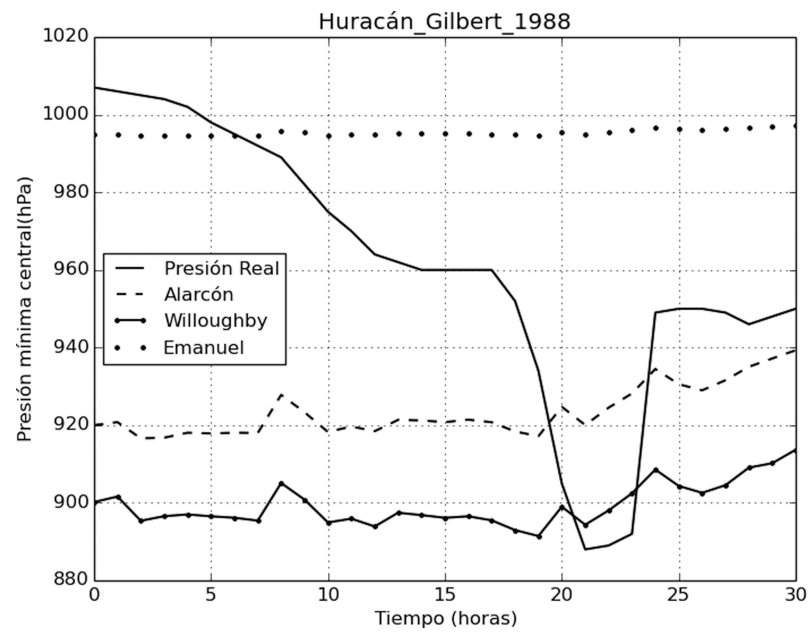

Figure 11: - Comparación entre la presión mínima real y la simulada (Gilbert) 


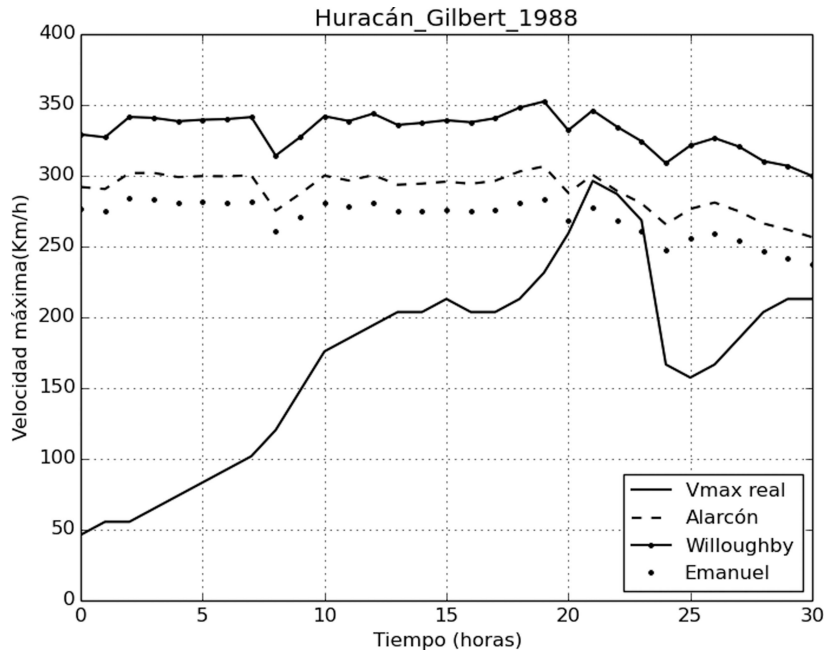

Figure 12: - Comparación entre la velocidad máxima del viento real y la simulada (Gilbert).

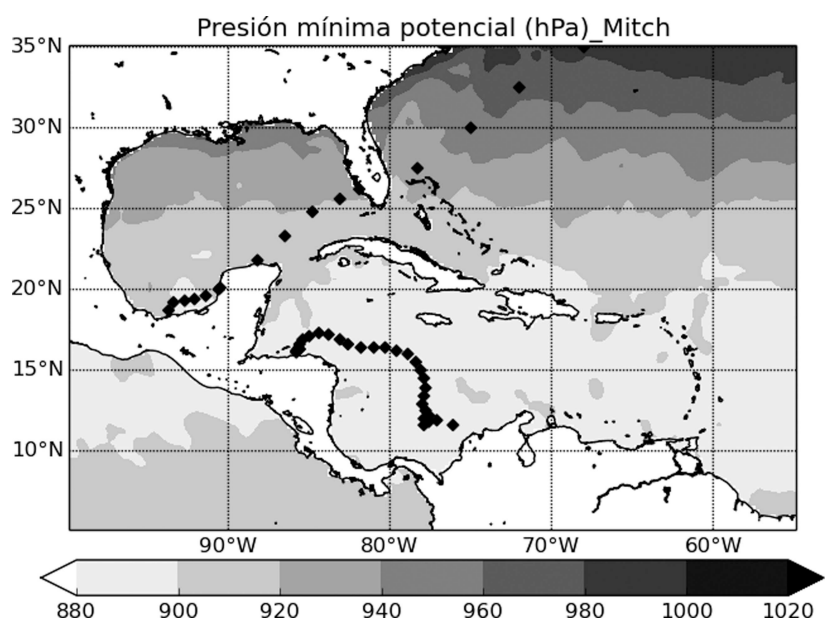

Figure 13: - Modelación de presión mínima central (Mitch).

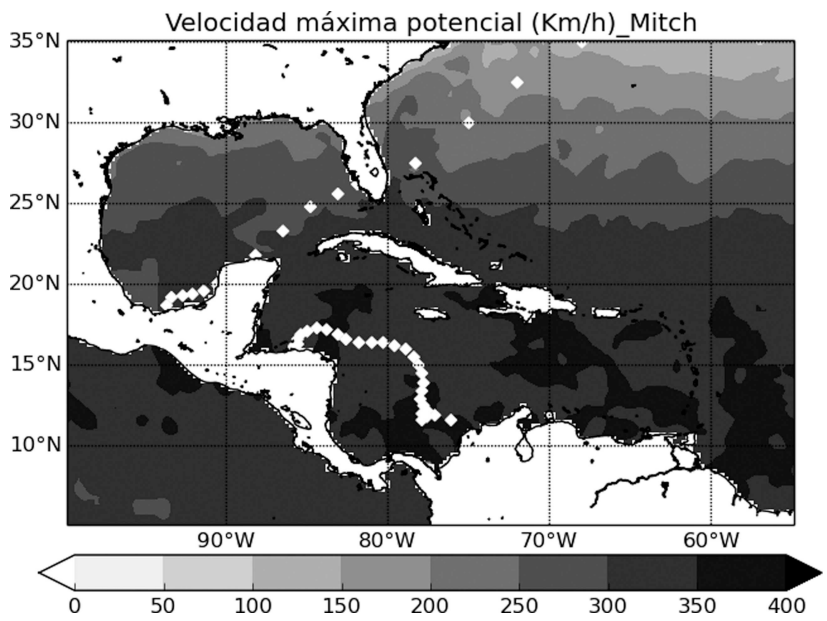

Figure 14: - Modelación de la velocidad máxima del viento (Mitch). la superficie. Aun así, el huracán Mitch alcanzó una presión mínima central de $905 \mathrm{hPa}$ y una velocidad máxima de los vientos de $287 \mathrm{~km} / \mathrm{h}$ (Alarcón, 2015). La simulación realizada para este sistema permite observar como el modelo calcula los valores de intensidad máxima potencial superior a la desarrollada por el huracán, existiendo convergencia en las curvas como muestran las Figs. 13 y 14. El modelo simuló una presión mínima central de $891 \mathrm{hPa}$ (Fig. 15) y una velocidad máxima del viento de $320 \mathrm{~km} / \mathrm{h}$ (Fig. 16).

\section{Conclusiones}

Se verificó el desempeño del modelo de intensidad potencial comparando los cálculos con datos reales de huracanes y se observó que para los huracanes más intensos de la cuenca atlántica simula la presión mínima central dentro de un rango máximo de incertidumbre de $6 \mathrm{hPa}$, lo

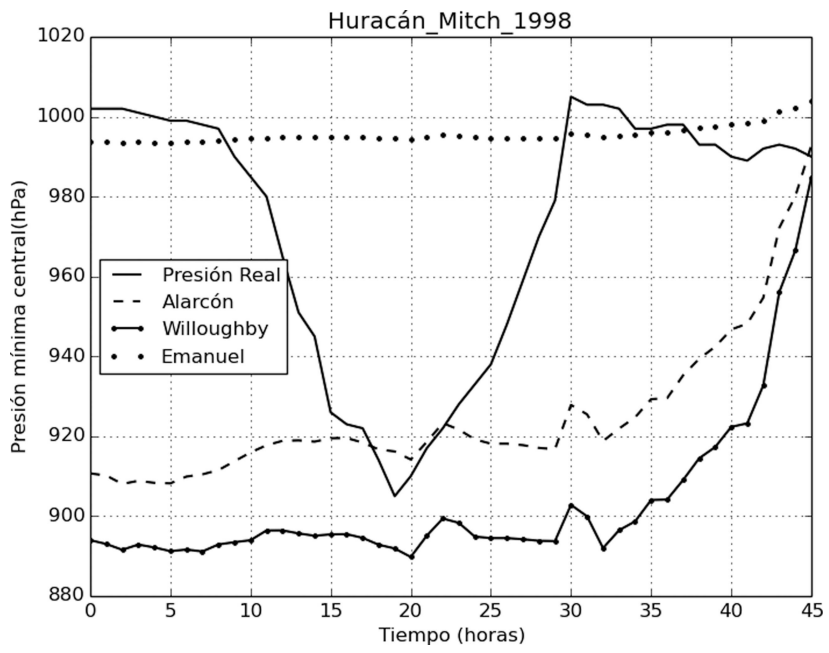

Figure 15: - Comparación entre la presión mínima real y la simulada (Mitch).

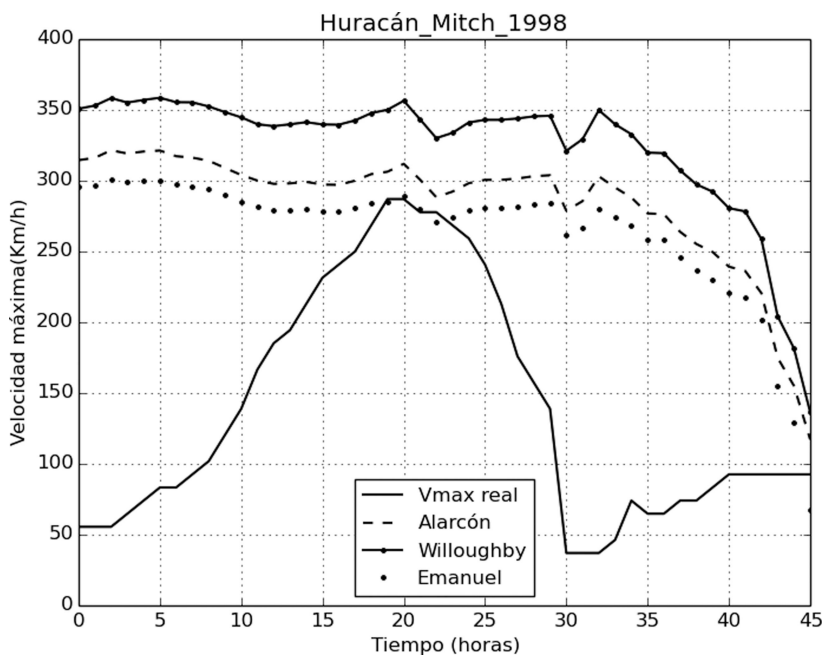

Figure 16: - Comparación entre la velocidad máxima del viento real y la simulada (Mitch). 
cual está relacionado con los cambios bruscos en el núcleo de la tormenta y en el poco intervalo de tiempo que ocurrieron. Para los menos intensos el comportamiento del modelo permitió comprobar la capacidad de este de simular la presión mínima central y la velocidad máxima del viento en superficie acercándose considerablemente a los valores reales.

En el cálculo de la velocidad máxima del viento se observaron algunos resultados por encima de los $100 \mathrm{~m} / \mathrm{s}$, lo que está influenciado por la temperatura de la tropopausa utilizada para las corridas del modelo que fue de $-73^{\circ} \mathrm{C}$, obteniéndose resultados similares a Wang et al. (2014) y por la presencia de una producción de una capa de espuma en la superficie del océano, permitiendo que ocurra una reducción del coeficiente de arrastre para velocidades por encima del umbral de $50 \mathrm{~m} / \mathrm{s}$ en la capa fronteriza (Golbraikh y Shtemler, 2006).

Se determinó un nuevo perfil radial de presión a partir del perfil de viento de Willoughby (2006), teniéndose consistencia física entre el cálculo de la presión mínima central y de la velocidad máxima del viento.

\section{Referencias}

ALARCON, A.P. Modelo para el cálculo de intensidad potencial de ciclones tropicales. VIII Congreso Cubano de Meteorología, Cuba, 2015.

BISTER, M.; EMANUEL, K. Dissipative heating and hurricane intensity. Meteorology and Atmospheric Physics, v. 65, n. 4-5, p. 230-240, 1998.

BRYAN, G.H.; ROTUNNO, R. Evaluation of an analytical model for the maximum intensity of tropical cyclones. Journal of the Atmospheric Sciences, v. 66, n. 10, p. 3042-3060, 2009.

CAMP, J.; MONTGOMERY, M. Hurricane maximum intensity: Past and present. Monthly Weather Review, v. 129, p. 1704-1717, 2001.

ELSBERRY, R.L.; HOLLAND, G.J.; GARRISH, H.; DEMARIA, M; GUARD C.; EMANUEL, K. Is there any hope for tropical cyclone intensity change prediction? A panel discussion. Bulletin of the American Meteorological Society, v.73, n. 3, p. 264-275, 1992.

EMANUEL, K. An air-sea interaction theory for tropical cyclones. Part I. Journal of the Atmospheric Sciences, v. 43, n. 6, p. 585-604, 1986.

FRISIUS, T.; SCGONEMANNN, D. The impact of gradient wind imbalance potential intensity of tropical cyclones in an unbalanced slab boundary layer model. Journal of the Atmospheric Sciences, v. 70, n. 7, p. 1874-1890, 2013.
GOLBRAIKH, E.; SHTEMLER, Y.M. Drag coefficient for the air-sea exchange: foam impact in hurricane conditions. https://arxiv.org/ftp/arxiv/papers/1410/1410.1061.pdf.

HOLLAND, G.J. An analytic model of wind and pressure profiles in hurricanes. Monthly Weather Review, v. 108, n. 8, p. 1212-1218, 1980.

HOLLAND, G.J. The maximum potential intensity of tropical cyclones. Journal of the Atmospheric Sciences, v. 54, n. 21, p. 2519-2541, 1997.

MONTGOMERY, M.; SMITH, R. Paradigms for tropicalcyclone intensification. Australian Meteorological and Oceanographic Journal, v. 64, p. 37-66, 2014.

POWELL, M.D.; UHLHORN, E.W.; KEPERT, J.D. Estimating maximum surface winds from hurricane reconnaissance measurements. Weather and Forecasting, v. 26, n. 3, p. 774-776, 2011.

SMITH, R.K. Lectures on tropical cyclones. https://www.meteo. physik.uni-muenchen.de/ roger/Lectures/Tropical_Cyclones/060510 tropical_cyclones.pdf, 2006.

SMITH, R.; MONTGOMERY, M. Balanced boundary layers used in hurricane models. Quarterly Journal of the Royal Meteorological Society, v. 134, n. 635, p. 1385-1395, 2008.

SMITH, R.K.; MONTGOMERY, M.T.; VOGL, S. A critique of Emanuel's hurricane model and potential intensity theory. Quarterly Journal of the Royal Meteorological Society, v. 134, n. 632, p. 551-561, 2008.

VOGL, S. Tropical cyclones boundary-layers models. https:// edoc.ub.uni-muenchen.de/10274/, 2009.

WANG, Y., and WU, C.-C. Current understanding of tropical cyclone structure and intensity changes - a review. Meteorology and Atmospheric Physics, v. 87, n. 4, p. 257-278, 2004.

WANG, S.; CAMARGO. S.J.; SOBEL, A.H., POLVANI, L.M. Impact of the tropopause temperature on the intensity of tropical cyclones: An idealized study using a mesoscale model. Journal of the Atmospheric Sciences, v. 71, n. 11, p. 4333-4348, 2014.

WILlOUGHBY, H.E.; DARLING, R.W.; RAHN, M.E. Parametric representation of the primary hurricane vortex. Part II: A new family of sectionally continuous profiles. Monthly Weather Review, v. 134, n. 4, p. 1102-1120, 2006.

\section{Direcciones de Internet}

NOAA, http://nomads.ncdc.noaa.gov/data.php?name=acces\#climatencdc, accedido en 4/4/2017.

HURDAT, http://www.aoml.noaa.gov/hrd/hurdat/Data_Storm. html, accedido en 14/4/2017.

This is an Open Access article distributed under the terms of the Creative Commons Attribution License, which permits unrestricted use, distribution, and reproduction in any medium, provided the original work is properly cited. 